\title{
Results of the Collaborative International Evaluated Library Organisation (CIELO) Project
}

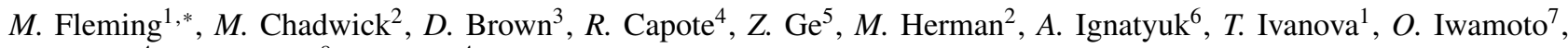 \\ $A$. Koning ${ }^{4}, A$. Plompen ${ }^{8}, A$. Trkov ${ }^{4}$, and all of the contributors to the CIELO Project \\ ${ }^{1}$ OECD Nuclear Energy Agency, Boulogne-Billancourt, France \\ ${ }^{2}$ Los Alamos National Laboratory, Los Alamos, NM, USA \\ ${ }^{3}$ Brookhaven National Laboratory, Upton, NY, USA \\ ${ }^{4}$ IAEA Nuclear Data Section, Vienna, Austria \\ ${ }^{5}$ China Institute of Atomic Energy, Beijing, China \\ ${ }^{6}$ Institute of Physics and Power Engineering, Obninsk, Russia \\ ${ }^{7}$ Japan Atomic Energy Agency, Tokai-mura, Japan \\ ${ }^{8}$ European Commission, Joint Research Centre, Geel, Belgium
}

\begin{abstract}
Simulation of nuclear systems requires complete data that represents the relevant nuclear physics. This requires many types of experimental measurements, theoretical physics, semi-empirical models and software systems, as well as experts to integrate and guide the process. This discipline is collectively known as nuclear data, and separate programmes within various European countries, the USA, Japan, Russia, and other OECD Nuclear Energy Agency (NEA) member countries have been operating for many decades. The NEA Working Party on International Nuclear Data Evaluation Co-operation (WPEC) exists to improve the quality and completeness of nuclear data by bringing together representatives of the major nuclear data evaluation projects of NEA member countries and selected Invitees. The Sub- and Expert Groups of the WPEC typically focus on specific technical topics, while the Collaborative International Evaluated Library Organisation Pilot Project (CIELO) was established to generate complete evaluations for a selection of the most important isotopes for criticality in nuclear technologies: ${ }^{235,238} \mathrm{U},{ }^{239} \mathrm{Pu},{ }^{56} \mathrm{Fe},{ }^{16} \mathrm{O}$ and ${ }^{1} \mathrm{H}$.

This project stimulated numerous activities, resulting in major contributions to the Special Issue of the Nuclear Data Sheets journal and the production of a suite of new nuclear data evaluations that have been incorporated in major nuclear data libraries ENDF and JEFF. The outcomes of these evaluations include significant harmonisation of discrepancies between the independent programmes, improvement in the performance for international standard nuclear criticality and neutron transmission benchmarks, complete uncertainties for nearly all parameters and the utilisation of modern data storage technologies. This work has leveraged the considerable, parallel experimental work in collecting improved experimental measurements to support nuclear data and highlighted high-priority areas for further study. A productive and durable framework for international evaluation has been established which will build upon the lessons learned. These will continue through new WPEC groups and a new IAEA evaluation network, which has been initiated in response to the success of the CIELO project.

This article summaries some performance feedback on the CIELO evaluations, including recent results, and will describe ongoing and future, planned CIELO-related collaborations to further advance our understanding.
\end{abstract}

\section{Introduction}

Formed in 1989, the Working Party on International Nuclear Data Evaluation Cooperation (WPEC) has brought experts in nuclear data together to co-ordinate activities in experiments, theory, modelling, evaluation and validation. All of the world's nuclear data programmes, including those from NEA member countries, China and the IAEA, pool their expertise to address the largest challenges in their field. WPEC operates by forming subgroups to study and advance the state-of-the-art through well-defined projects over a (typically) three-year period.
Established as WPEC Subgroup 40, The Collaborative International Evaluated Library Organisation (CIELO) Project was an ambitious effort to produce international joint evaluations on the most essential isotopes: ${ }^{235} \mathrm{U}$, ${ }^{238} \mathrm{U},{ }^{239} \mathrm{Pu},{ }^{56} \mathrm{Fe},{ }^{16} \mathrm{O}$ and ${ }^{1} \mathrm{H}$. Due to their importance, the evaluation of these isotopes is a continuous challenge. Before CIELO, the performance of well-known libraries was already at an impressive level, but it was argued that by bringing together all of the WPEC participants, new theory and models, the most recent experimental data and sophisticated integral feedback methods, a three-year project could deliver evaluations with performance superior to the previous ENDF/B and JEFF releases.

*e-mail: michael.fleming@oecd-nea.org 
This Subgroup integrated findings from other WPEC Subgroups and spurred an intense collaboration, involving experts from over 70 institutions, that ultimately delivered as intended [1, 2]. New CIELO evaluations for each of these isotopes have been adopted in the new ENDF/BVIII.0 [3], JEFF-3.3 [4] and TENDL-2017 [5] libraries. A follow-up activity, focusing on multiple isotopes, including the suite of plutonium, has been started at the IAEA as the International Nuclear Data Evaluation Network (INDEN) that operates as a more loosely connected joint project in the spirit of CIELO. A new Subgroup on reproducibility has been established to work hand-in-glove with INDEN and all member country projects by integrating the wealth of NEA-coordinated validation expertise, NEA tools such as NDaST and the new collaborative NEA GitLab platform.

\section{Experimental data}

One of the guiding principles of the CIELO project was the direct engagement and utilisation of cutting-edge experimental techniques and data. There is no substitute for quality differential data and this provides the basis of an improved, general-purpose evaluation. Results in comparisons against integral data, including criticality benchmarks, provide many of the performance criteria that are used to judge evaluations. Impressive progress in integral assimilation methods [6] and the development of automated sensitivity-based tools such as the NEA NDaST [7] provide focus points for evaluators, while differential experiments provide the primary evidence for nuclear data evaluation. The list of measurements ${ }^{1}$ performed during, and used in, the CIELO project are listed in Table 1. An example is shown in Figure 1, where the availability of new measurements (then preliminary results) directed the new ${ }^{239} \mathrm{Pu}$ capture evaluation. Although the Subgroup was completed, with the delivery of final files and reports, many of these experimental campaigns have continued without pause, acquiring and analysing new data ${ }^{2}$ on the CIELO isotopes and others.

In addition to these experiments, the CIELO project established a consensus on the adoption of the IAEA Neutron Data Standards [10], which represents a detailed, parallel evaluation. The Standards working group not only considered many experimental sources, but re-evaluated uncertainties in experiments. This resulted in a doubling of standard fission cross section uncertainties due to previously unrecognised systematic uncertainties.

\section{New evaluations}

An actinide evaluation contains numerous types of data, ranging from cross sections and resonance parameters to emitted particle spectra and delayed particle emission probabilities. Each evaluated file must not only carefully

\footnotetext{
${ }^{1}$ For a list of references, please see [2].

${ }^{2}$ Indeed, some of the data used in the CIELO evaluations were preliminary, with data still in the process of being finalised and submitted to EXFOR [8].
}

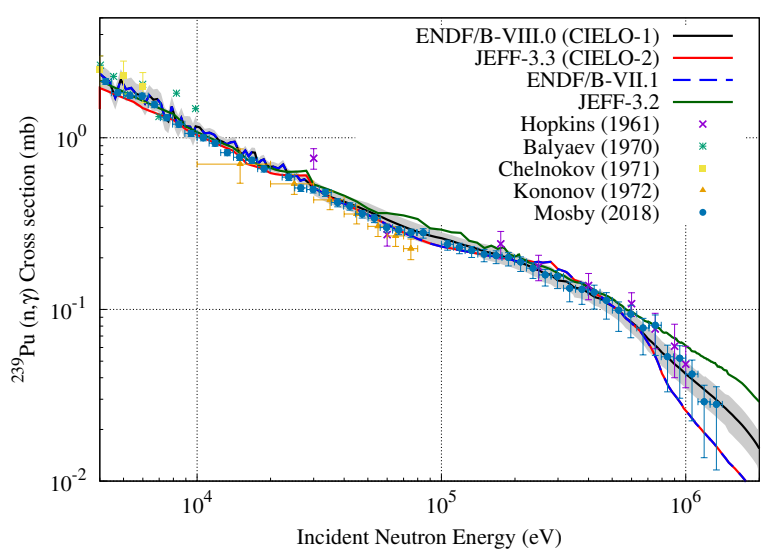

Figure 1. The CIELO evaluations for ${ }^{239} \mathrm{Pu}$ neutron capture, including data from new measurements at the LANL DANCE [9]

describe each of these aspects of the physics, but balance each against other correlated phenomena (and the effects of other isotopes when considering integral performance). Each isotope (c.f. [11]), and in some cases even components of files (c.f. [12]) require their own full-length publications to describe. Below we provide a brief overview of major outcomes.

\subsection{Fission in ${ }^{235} \mathrm{U}$ and ${ }^{239} \mathrm{Pu}$}

The re-evaluation of the IAEA Neutron Standards included a revision of the fission cross section uncertainties due to previously unrecognised uncertainties. These have a significant impact on the propagation of nuclear data uncertainties into critical benchmarks, providing a more credible estimate of the true uncertainties based on differential data [11].

New measurements [13] supported a softening of the prompt fission neutron spectra for ${ }^{235} \mathrm{U}$ for incident energies below $2 \mathrm{MeV}$. This also directly affects the ratio of PFNS, making ${ }^{239} \mathrm{Pu}$ relatively 'hotter' than ${ }^{235} \mathrm{U}$, as shown in Figure 2. This is best seen in the mixed metal fast systems, which had an average improvement by over $100 \mathrm{pcm}$ in integral testing by S. van der Marck of the NRG $[2,14]$.

\subsection{Capture and scattering in ${ }^{235,238} \mathrm{U}$ and ${ }^{239} \mathrm{Pu}$}

The new Neutron Standards have utilised several more recent ${ }^{238} \mathrm{U}$ neutron capture measurements and these have been directly incorporated into the CIELO evaluations. New JRC-Geel resonance evaluations at lower energies used measurements from GELINA and ORNL. Experimental evidence from RPI led to changes in the ${ }^{235} \mathrm{U}$ capture cross sections, with a decrease near $1 \mathrm{keV}$ and an increase near $10 \mathrm{keV}$. Future experiments are planned to corroborate these changes.

Neutron scattering evaluations have benefitted from a novel semi-differential technique employed at RPI that, coupled with modelling improvements, resulted in a reevaluation of ${ }^{238} \mathrm{U}$ inelastic scattering. Additional mea- 
Table 1. Notable experimental contributions made during the CIELO project

\begin{tabular}{l|l}
\hline Laboratory & Measured data relevant for CIELO \\
\hline CERN n_TOF & $\begin{array}{l}{ }^{235,238} \mathrm{U} \text { fission cross sections } \\
{ }^{235,238} \mathrm{U} \text { neutron capture cross sections }\end{array}$ \\
\hline JRC-Geel & ${ }^{238} \mathrm{U}$ neutron capture cross section \\
& Fe inelastic scattering cross section \\
& ${ }^{16} \mathrm{O}(\mathrm{n}, \alpha)$ cross section
\end{tabular}

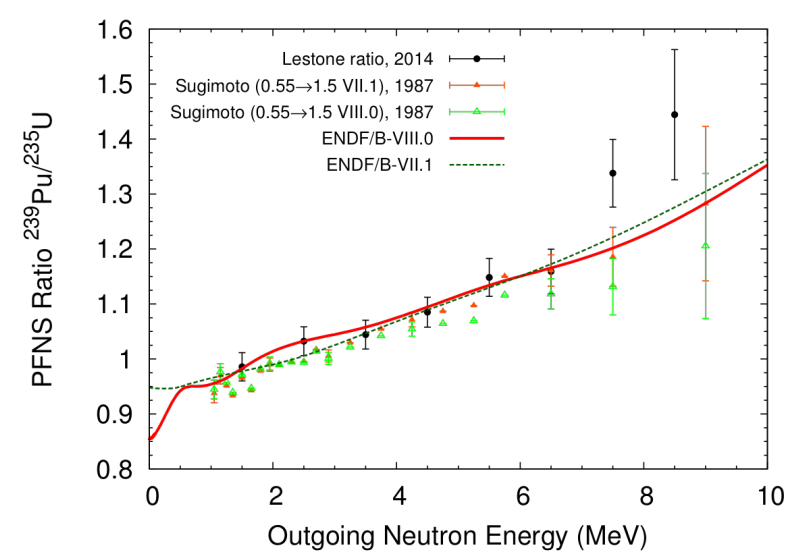

Figure 2. Ratio of the probabilities of prompt fission neutron energy emission as a function of outgoing energy between ${ }^{239} \mathrm{Pu}$ and ${ }^{235} \mathrm{U}$ with $1.5 \mathrm{MeV}$ incident neutrons

surements are planned for ${ }^{235} \mathrm{U}$ and ${ }^{239} \mathrm{Pu}$ to inform similar re-evaluations.

\subsection{Evaluation of ${ }^{56} \mathrm{Fe}$}

The evaluation of iron includes a resonance evaluation up to the first inelastic level at $847 \mathrm{keV}$, which is followed by resonating cross sections up to around $2 \mathrm{MeV}$. New semi-differential data from RPI, as with ${ }^{238} \mathrm{U}$ was used to perform this component of the evaluation. As in previous evaluations, the addition of a background cross section is required above $400 \mathrm{keV}$ and, in the CIELO- 1 evaluation, a background was added to extend the characteristic thermal $1 / v$ shape up to $10 \mathrm{keV}$. These are partly driven to address issues found with integral benchmark studies and are responsible for some of the improved performance of both ENDF/B-VIII.0 and JEFF-3.3 in integral benchmarks [15].
Inelastic scattering above $4 \mathrm{MeV}$ remained a complex issue during CIELO, with normalisation issues being raised in some of the most recent data from JRC-Geel. Although it was believed to have been resolved, feedback since the release of the CIELO files, the ENDF/B-VIII.0 and JEFF-3.3, notably including shielding studies with macroscopic quantities of iron, have shown that further study is needed.

\subsection{Evaluation of ${ }^{16} \mathrm{O}$}

Following many years of advocating for a revision of the ${ }^{16} \mathrm{O}(n, \alpha)$ cross section, R-matrix experts re-evaluated this channel with a $40 \%$ increase in the $3-6 \mathrm{MeV}$ range. This was supported by a detailed analysis of inverse and thick target yield data. New measurements are planned to corroborate this change. For the total cross section, disagreements between the Cierjacks 1968 and 1980 data, amongst others, left open some uncertainty in the total normalisation. New RPI measurements using a novel $2.3 \mathrm{MeV}$ oxygen window technique provided the needed confirmation, remarkably agreeing with the new CIELO-1 evaluations, which were completed before the measurements, within $1 \%$. Many thermalised oxygen-containing benchmark are highly sensitive to the thermal elastic scattering cross section of ${ }^{16} \mathrm{O}$. Following an analysis by Kopecky and Plompen, the CIELO-2 evaluation adopted a value of 3.765, while the previous ENDF/B-VII.1 was reduced by $1.5 \%$ for CIELO- 1 (worth more than $150 \mathrm{pcm}$ in many solution benchmarks) and brought the two values within $1 \%$ of each other.

\subsection{Final CIELO file suites}

It must be noted that experimental uncertainties provide evaluators with a space of possible vales that are all be equally valid. Each nuclear data projects must support, 
Table 2. The lead institutes for the evaluation of both CIELO suites, with resonance and fast incident-energy ranges separated

\begin{tabular}{l|l|l}
\hline Evaluation & CIELO-1 & CIELO-2 \\
\hline${ }^{1} \mathrm{H}$ & LANL/IAEA & LANL/IAEA \\
\hline${ }^{16} \mathrm{O}$ res. & LANL/JRC-Geel & IRSN/JRC-Geel \\
\hline${ }^{16} \mathrm{O}$ fast & LANL & LANL \\
\hline${ }^{56} \mathrm{Fe}$ res. & IAEA/BNL & IRSN \\
\hline${ }^{56} \mathrm{Fe}$ fast & BNL/IAEA/CIAE & JEFF \\
\hline${ }^{235} \mathrm{U}$ res. & ORNL/IAEA & IRSN/ORNL \\
\hline${ }^{235} \mathrm{U}$ fast & IAEA+LANL(PFNS) & CEA \\
\hline${ }^{238} \mathrm{U}$ res. & JRC-Geel & IRSN/CEA \\
\hline${ }^{238} \mathrm{U}$ fast & IAEA+LANL(PFNS) & CEA \\
\hline${ }^{239} \mathrm{Pu}$ res. & ORNL/CEA & ORNL/CEA \\
\hline${ }^{239} \mathrm{Pu}$ fast & LANL & CEA \\
\hline
\end{tabular}

develop and use their own models and tools to create final evaluations that meet the needs of their stakeholders. The CIELO project achieved considerable harmonisation in the adoption of Neutron Standards and resolution of several outstanding discrepancies. However, the goal of the CIELO project was not to generate one monolithic evaluation. As the project completed, two different suites of files were found to have excellent performance in validation exercises (as discussed in Section 4).

To accommodate the two, self-consistent evaluated file sets that emerged from the CIELO activities, two versions were issued. These were labelled CIELO-1 and CIELO-2 and were later adopted by the ENDF/B-VIII.0 and JEFF3.3 libraries, respectively. Table 2 summarises the leading institute(s) that evaluated the main components for each file in both of the CIELO versions. It should be noted that these versions do not represent two evaluations from two segregated communities. Files from the US Oak Ridge and Los Alamos National Laboratories appear in CIELO2 and files from the EU Joint Research Centre Geel and the French Alternative Energies and Atomic Energy Commission (CEA) appear in CIELO-1. Indeed, some results from participants in the JEFF community were selected for CIELO-1 and not for CIELO-2.

\section{Results with integral benchmarks}

Integral benchmarking provides feedback on the entirety of a library and how it performs in scenarios of interest to applications, such as the simulation of critical systems. Through validating the results of simulations against respected integral benchmarks, such as those contained with the International Criticality Safety Benchmark Evaluation Project (ICSBEP) [16], confidence can be established in the use of a library for applications similar to those benchmarks. The standard metric for testing sets of integral results is the reduced chi-squared,

$$
\chi^{2}=\frac{1}{n} \sum_{i=1}^{n} \frac{\left(C_{i} / E_{i}-\mu\right)^{2}}{\sigma_{i}^{2}}
$$

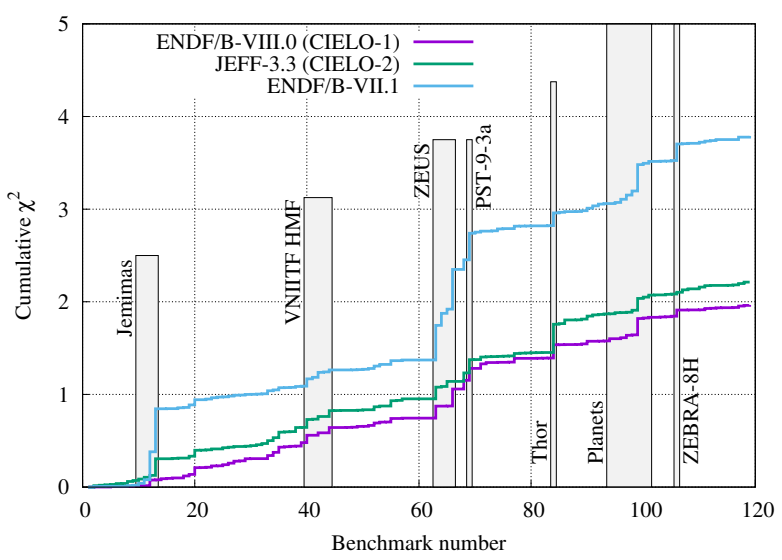

Figure 3. Cumulative, reduced chi-squared values for the Mosteller suite of criticality benchmarks

where the calculated and experimental values $C_{i}$ and $E_{i}$, subject to the experimental uncertainties $\sigma_{i}$, are compared with the averaged value $\mu$. The reduced chi-squared can be used to test whether the calculated values for a set of benchmarks are different from the experimental values in a statistically significant way. In practice, this identifies benchmarks with significant discrepancies and evaluation projects must identify ways to correct these while maintaining the agreement found on the majority of wellperforming cases.

There are well over 4000 cases in ICSBEP and thousands were simulated during the CIELO project. Drawing meaningful conclusions from such data is often challenging, due to the sheer size of the information as well as the unquantified correlations between experiments and few benchmarks with suspiciously low uncertainties. Carefully selected suites of benchmarks, such as the Mosteller suite, are used to cover essential physics while minimising computational burden. The results for this suite are shown in Figure 3. The small subset of problematic cases for ENDF/B-VII.1, including the Jemimas (fast metal with intermediate-enriched uranium) and ZEUS (intermediate spectrum, metal with highly-enriched uranium) provide half of the cumulative, reduced chi-squared. Both CIELO-1 and CIELO-2 improved the agreement on these benchmarks while maintaining performance on the others against the ENDF/B-VII.1 and JEFF-3.2 libraries.

\section{Future}

As shown in Figure 4, CIELO directly precipitated the most recent major nuclear data libraries. The areas that CIELO made progress on are continuing on virtually all fronts. More measurements are ongoing or planned to apply the facilities and techniques mentioned above to reaction channels in other isotopes, such as the continuing Chi-Nu experiments or RPI semi-differential measurements. These will continue to be shared through the IAEA International Nuclear Data Evaluation Network (INDEN) and WPEC as the evaluation projects maintain the co- 


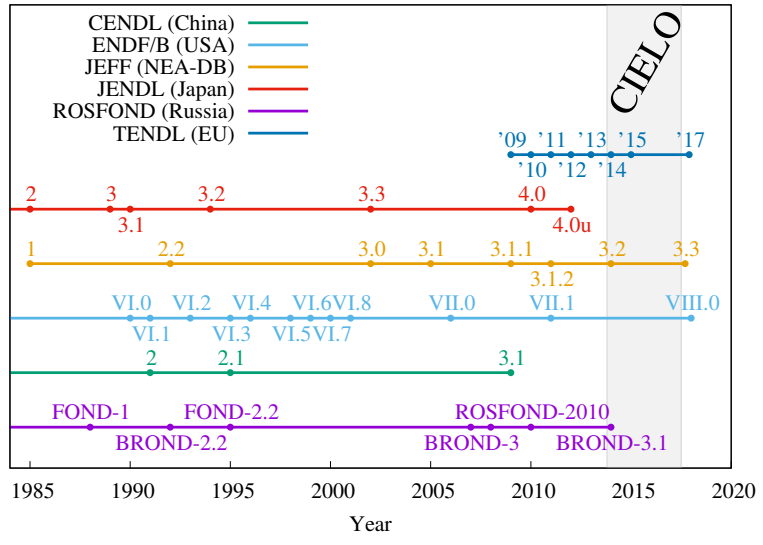

Figure 4. History of nuclear data libraries and their releases

operation that enabled CIELO to successfully deliver on its goals.

Even before the CIELO files were released through the ENDF/B-VIII.0 and JEFF-3.3 libraries, the community of users and evaluators were already probing the data, highlighting differences and looking for areas where we can further harmonise our data [17]. Precisely through these and other efforts, we have a clear agenda for INDEN and have already understood some of the outstanding challenges, such as iron inelastic scattering and its impact on shielding benchmarks.

The fact that both CIELO-1 and CIELO-2, with their various differences, were able to make substantive improvement on the same benchmark suites highlights the fact that we can not yet restrict our knowledge of physics into only one evaluation. Through co-operation and coordinated projects, we demonstrated that it is possible to constrain the space of possible evaluations, obtain better agreement with existing and new experimental data, and find solutions that meet the needs of our end-users.

\section{References}

[1] OECD-NEA, International Co-operation in Nuclear
Data Evaluation: An Extended Summary of the Collaborative International Evaluated Library Organisation (CIELO) Pilot Project, WPEC Subgroup 40, NEA Publication No. 7498 (2019)

[2] M. Chadwick et al., Nuclear Data Sheets 148, 189 (2018), special Issue on Nuclear Reaction Data

[3] D. Brown et al., Nuclear Data Sheets 148, 1 (2018), special Issue on Nuclear Reaction Data

[4] A. Plompen et al., The Joint Evaluated Fission and Fusion Nuclear Data Library, JEFF-3.3, in preparation

[5] A. Koning, D. Rochman, J.C. Sublet, N. Dzysiuk, M. Fleming, S. van der Marck, Nuclear Data Sheets 155, 1 (2019), special Issue on Nuclear Reaction Data

[6] M. Salvatores et al., Nuclear Data Sheets 118, 38 (2014)

[7] J. Dyrda, I. Hill, L. Fiorito, O. Cabellos, N. Soppera, EPJ Nuclear Sci. Technol. 4, 14 (2018)

[8] N. Otuka et al., Nuclear Data Sheets 120, 272 (2014)

[9] S. Mosby et al., Nuclear Data Sheets 148, 312 (2018), special Issue on Nuclear Reaction Data

[10] A. Carlson et al., Nuclear Data Sheets 148, 143 (2018), special Issue on Nuclear Reaction Data

[11] R. Capote et al., Nuclear Data Sheets 148, 254 (2018), special Issue on Nuclear Reaction Data

[12] D. Neudecker, et al., Nuclear Data Sheets 148, 293 (2018), special Issue on Nuclear Reaction Data

[13] M. Devlin et al., Nuclear Data Sheets 148, 322 (2018), special Issue on Nuclear Reaction Data

[14] S.C. [van der Marck], Nuclear Data Sheets 113, 2935 (2012), special Issue on Nuclear Reaction Data

[15] M. Herman et al., Nuclear Data Sheets 148, 214 (2018), special Issue on Nuclear Reaction Data

[16] OECD Nuclear Energy Agency, ICSBEP Handbook 2018, DOI:10.1787/ea7c647e-en

[17] R. Capote, A. Trkov, EPJ Nuclear Sci. Technol. 4, 27 (2018) 